\title{
Measurement, error, information, and interpretation
}

\author{
Zeevi Dvir https://www.mendeley.com/authors/7005648179
}

In a few month time one of the corner stones of classical metrology (the science of measurement) will become obsolete serving from that point on as a museal artefact but one of an immense scientific value. The standard kilogram (SK), is located and protected under the strictest conditions at the International Bureau of Weights and Measures, in St. Claud, near Paris. For the last 130 years the SK was the ultimate yardstick for the mass of every other measurable object but in May 2019 it will be replaced by a complex derivative of the Planck Constant, a new standard of mass. The main reason for this transformation has been the discovery that the SK has gained tens of micrograms of mass from surface contamination and hence its allegedly identical copies, which are placed at various national institutions in the world, are no longer of an exactly identical mass. This disparity leads first and foremost to the apparent loss of standardization but at the same means that the SK or its copies can no longer be held as the absolute standard, a situation which for some physics applications is apparently of high significance. And along with the SK some other 'absolutes' will come down like those for time and distance. They are all going to be replaced by other 'more absolute absolutes.' Indeed, if we admit now that the former absolute can no longer be considered as a true absolute, and that differences, perhaps individual, between the SK and each of its 'identical twins' exist, we confess to a certain error that is integral to all measurements. Furthermore, although it may seem that the new standards belong exclusively in the arcane world of physical metrology, they will, in due course, affect the way we measure and interpret such quantities as time, mass and distance, which ultimately will gravitate into measurements applied in the biomedical domains.

But this is still a distant echo since as clinicians the measure- ments we take and the errors we are accustomed to, even unconsciously, are of a different order of magnitude altogether. So how the error should be defined? Relating to the difference between the true and actual value of the measured variable, error refers to the cluster of factors that make up this difference. And as these factors may change with time, so will the error. Therefore, since the concept of error is eminently important in the clinical domain, this editorial presents in a short and rather simplified way its meaning and implications.

We 'measure' from the very beginning of our life. Measurement is a process of comparison, namely a given quantity or trait is set against a standard and some kind of a 'judgment' is made. Take for example individual sensation, irrespective of the modality: vision, audition, touch etc. The process of 'judging' is based on comparison of the sensed modality against or according to some reference that may be either internal or external, innate or acquired. Thus, focusing on the sense of taste, it is there even before the baby is born and bitterness, among the various individual 'master tastes' is well reacted to when some kind of a bitter food is brought to the baby's mouth. This reaction is at least partly, a product of comparison against some internal standard, as bitterness can be graded according to some scale, inasmuch as sweetness or sharpness are. And as we grow, we employ our 'measurement' skills in challenging domains outside the sensory world like various cognitive situations and/or emotional conditions, whereby we embrace the relative nature of things, physical as well as abstract.

In some respect, this extraordinary process of comparison, the act of 'measurement', assumes its full volume when one becomes a professional, particularly in those cases where, what we understand 
by the terms of measuring and measurement (when applied in the narrower sense), is an integral part of one's 'practice,' be it related to science, pure and applied, engineering, or the various branches of bio-medicine. Among the latter, clinical measurements relating to human beings rather than to animals or microorganisms are of particular interest to the readership of this journal. Significantly, most of the clinical of measurements relate to variables that largely do not require our active participation while remaining largely beyond our ability to exert volitional control over. Examples for such variables range from biochemical markers in the blood or urine, genetic and pathological tissue markers, radiological findings and up to the more physiologically oriented such as the electrophysiological or mechanical (e.g., blood flow). Characterized by what may be described as having sufficient accuracy, stability and responsiveness, these measurements, when correctly applied and interpreted can lead to the correct diagnosis, still the stronger part of medicine, and to planning the optimal intervention.

In rehabilitation, measurements take a different perspective due to the involvement of a critical element that does not exist in the bio-medical domain at large. Here, the measured entity is invariably the performing patient and therefore as the measured variables are performance-related, they are affected by the patient. Moreover, they may be equally affected by another performer - the examiner, and still further by the measurement tool and the test conditions.

Imagine for example the need to measure the range of motion (RoM) of the head (cervical spine) in a patient, by the same clinician, at the day of the measurement, is suffering from low back pain. Although the latter should generally not affect cervical motion, it is not impossible that the recorded RoM will in fact be smaller than what would have been the case under a pain-free low back. Indeed, if on the following measurement the RoM increases, this would not necessarily indicate improvement in cervical motion as much as a reflection of the error i.e., a change within the patient that was not related to the actual measurement. In this case one deals with a patient-related error. Consider now the situation where cervical RoM is successively measured (e.g., with a difference of $15 \mathrm{~min}$ ) by two different clinicians. How are we to interpret a difference in measurement, if such is obtained? Unless some dramatic and unexpected change took place within such a short span, the existence of an error is indicated, this time of the examiner-related type. However, who among the two is the reference, or in other words, whose test findings should be referred to as the 'true' finding? Although we might be enticed into taking the average value of the measurements, there is no 'correct' answer to this problem.
Things may get even more involved and often confusing, when rather than employ a physical measurement tool, use is made of questionnaires or scales whose individual ranks may not be sensitive enough to the problem at hand or might fluctuate on repetitive measurements due to intervening factors that are not related to the clinical issue. Thus the presence of an error is an inevitability. Errors are companions of every measurement process and it is particularly significant in the study of human performance, and most critically so when the performance relates to patients, rather than their healthy counterparts.

For this reason, statistical methods which embrace the concept of error have been devised and been put to use, increasingly so in the recent decade. These methods allow us, to a great extent, to judge whether changes we observe in the level of human performance are merely within the error or outside it. In the former case, the change should not be regarded as associated with a meaningful clinical change (MCC), and should therefore be ignored. On the other hand, if the change is sufficiently large so that it is outside the research-based calculation of the domain of the error, it may be regarded as indicating an MCC. The scientific and professional literature now abound with studies relating to MCC and practitioners of clinical rehabilitation and exercise intervention, should be well aware of this emerging and most important concept.

We measure in order to be informed. But information on its own is not enough although it is absolutely essential. What we do with the information, namely the act of interpretation, is really what matters at the end of the day. One important cornerstone of interpretation is recognizing the specific weight of the error in the context of measurement. In other words, what is significant, and what is not. Therefore, while without measurement we cannot discharge our responsibility in a professional manner, ignorance of the error is equally irresponsible, and may lead to erroneous decisions, regarding the level of the patient (status) as well as his progression/stagnation/deterioration during rehabilitation.

\section{CONFLICT OF INTEREST}

No potential conflict of interest relevant to this article was reported.

Editor of the Journal of Exercise Rehabilitation Department of Physical Therapy, Steyer School of Health Professions, Sackler Faculty of Medicine, Tel Aviv University, Tel Aviv 69978, Israel E-mail: zdvir@post.tau.ac.il 